\title{
Sports dermatology part 1: common dermatoses
}

Q ports and physical exercise $\checkmark$ have become integral to modern life. Although their benefits are plentiful, participation in athletic activities can also be associated with a wide variety of skin problems. Sports dermatoses can result from mechanical trauma, exposure to environmental and infectious agents and various other factors. The diagnosis of specific sports dermatoses can at times be challenging and requires familiarity with their characteristic clinical presentations. In this article we highlight some of the common skin problems associated with athletic activities.

\section{Anatoli Freiman}

Division of Dermatology

$\approx$ McGill University

Montréal, Que.

Benjamin Barankin

Division of Dermatology

University of Alberta

Edmonton, Alta.

David J. Elpern

The Skin Clinic

Williamston, Mass.
Acne mechanica: Commonly referred to as sports-induced acne, acne mechanica is caused by a combination of heat, pressure, occlusion of the skin and repetitive frictional rubbing. It usually occurs in areas covered by protective gear in contact sports. For instance, football players often have follicular papules, pustules and nodules on the forehead, preauricular areas, chin and shoulders where these touch the helmet and straps.

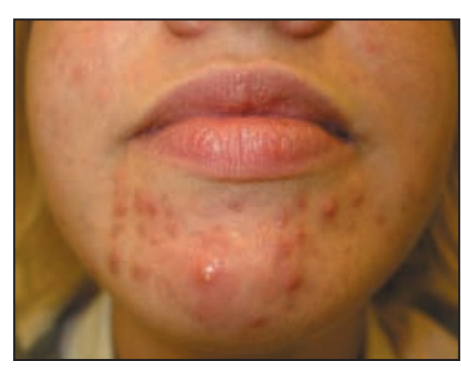
The equestrian shown in the figure has acne mechanica resulting from contact with her chin strap. Golfers can experience acne mechanica on the lateral back where the strap of their golf bag rubs. Allergic contact dermatitis caused by substances found in sports equipment (e.g., rubber chemicals such as thiurams, mercaptobenzothiazole and carbamates) needs to be considered in the differential diagnosis of acne mechanica. The former appears more eczematous and pruritic and can be confirmed by specialized patch testing.

Management: In addition to preventive measures, the treatment of acne mechanica is similar to that of other forms of acne, using topical or systemic antibiotics, benzoyl peroxide and topical retinoids.

Foot blisters: Shearing forces during exercise, compounded by perspiration, can separate the layers of the epidermis and lead to friction blisters, which are often quite painful and debilitating. New or poorly fitted footwear is a frequent culprit.

Management: Properly fitting shoes and socks worn without folds and creases, as well as frequent sock changes in order to avoid moisture accumulation, are cornerstones of blister prevention. Socks made of synthetic materials (e.g., acrylic) may help to collect moisture and keep it away from the skin. Antiperspirant agents containing aluminum chloride applied to the bottom of the feet before athletic activity may be effective in reducing foot blisters. In a double-blind trial in which military cadets applied either an antiperspirant containing 20\% aluminum chloride or a placebo to their feet for at least 3 nights before a long hike, the rate of foot blisters in the group using the antiperspirant was only $21 \%$, compared with $48 \%$ in the placebo group $(p<0.01) .{ }^{1}$ How-

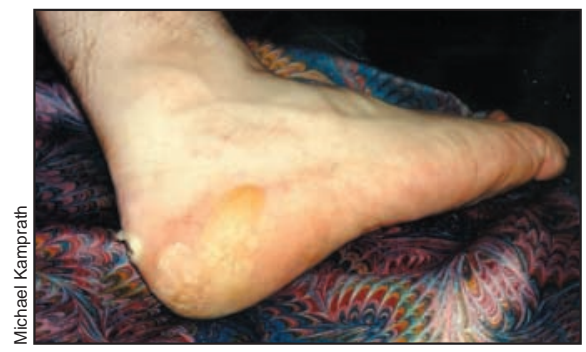
ever, the cadets who used the antiperspirant were also significantly more likely to experience foot irritation (57\% v. $6 \%, p<0.01)$. Toe padding may also prevent blistering. Once blisters form, it is generally recommended that small ones (diameter $<1 \mathrm{~cm}$ ) be left intact and large ones drained using an aseptic technique. The blister roofs should be left in place as a protective covering in order to accelerate healing. Moleskin tape, which is available over the counter, can be used in the form of a "blister doughnut" that is applied over the area. 
Black heel/talon noir: This black discolouration of the posterolateral aspects of the heel is caused by trauma-induced hemorrhage in an area where blood vessels are minimally protected by the overlying skin. Black heel is associated with frequent quick starts and stops, motion that is typical of tennis or basketball, but it can also occur in football and other

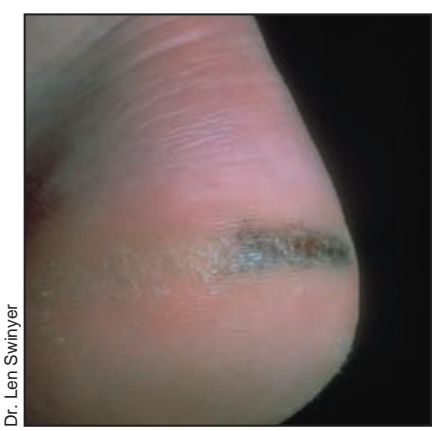
sports. Black palm, or tache noir, refers to punctate hemorrhages seen in athletes who apply pressure to their hands, such as racquet sports players, weightlifters, gymnasts and golfers. Such lesions must be differentiated from acrolentiginous melanoma. This can be done by gently paring the superficial skin layers of the affected area with a scalpel blade: the black colour can be pared away in black heel or black palm, but the deeper pigment in melanoma usually cannot be removed with this method.

Management: Both black heel and black palm are harmless and usually resolve with time.

Jogger's toe/tennis toe: Trauma-induced subungual or periungual hemorrhage is common among athletes. When toenails slide forward repetitively, colliding with the front of the shoes, this may lead to hematoma formation, onycholysis and subungual hyperkeratosis. The black-blue discolouration is frequently observed under the distal nail plate, sparing the proximal nail.'2 Specific mechanical stresses in various sports may lead to different nails being affected. Similar hemorrhages can occur in workers who wear steel-

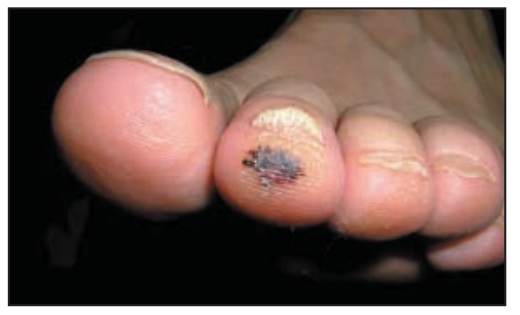
toed shoes or boots. The differential diagnosis includes subungual melanocytic nevus or malignant melanoma, and biopsy of the nail bed may be warranted, especially if the discolouration is unilateral or extends to the nail fold (known as Hutchinson sign).

Management: Properly fitted shoes and orthotic devices may help prevent the forefoot from slamming into the toe box. If a patient presents with an acute subungual hemorrhage, drainage with a needle or a heated paperclip through the nail plate can quickly reduce local pressure and discomfort.

Piezogenic pedal papules: These skin-coloured or yellowish papules along the lateral plantar surface of the heels may become obvious upon prolonged standing or exercise (the figure on the left shows papules before exercise; the figure on the right, papules after exercise). They are common among long-distance runners but can occur in non-athletes as well, particularly obese people. Herniation of subcutaneous fat is thought to occur through small tears in the plantar fascia. The lesions are occasionally painful, and elevation of the feet often provides relief.

Management: Although no successful treatment has been found, heel cups inserted into shoes can often alleviate the discomfort during exercise. ${ }^{2}$
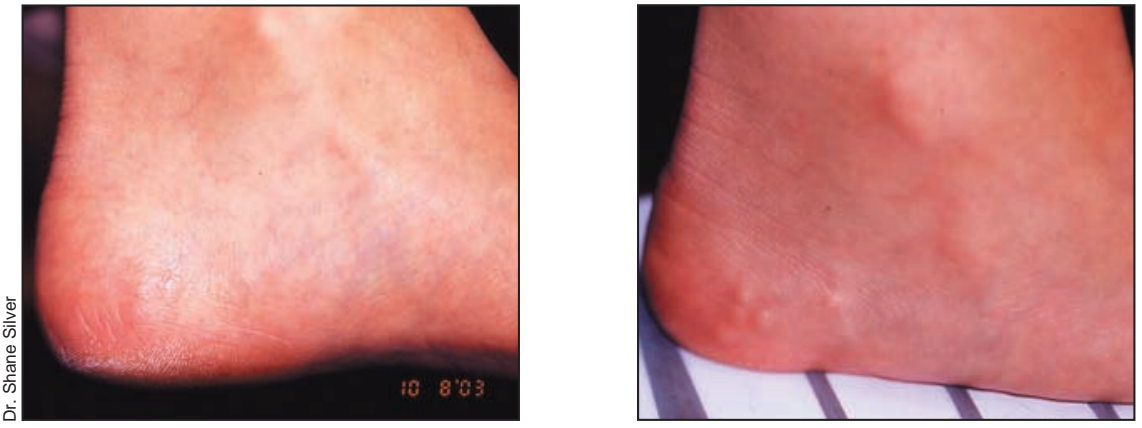
Pityrosporum folliculitis: This common fungal infection of athletes is caused by the overgrowth of Pityrosporum orbiculare yeast. Often misdiagnosed as bacterial folliculitis or acne vulgaris, it presents with erythematous follicular papules or pustules on the upper back, trunk and shoulders. In contrast to acne, these patients often do not have pronounced facial lesions. Pityrosporum folliculitis often occurs in the setting of heat, sweating and poor hygiene.

Management: Ketoconazole 2\% or selenium sulfide 2.5\% shampoos applied twice weekly (leaving on for 10-15 minutes) for 2-4 weeks can be used. In our experience, however, oral antifungal agents (itraconazole or ketoconazole) are the therapy of choice, since topical therapy is often ineffective. The rate of recurrence is very high among athletes, and prophylactic use of $2 \%$ ketoconazole shampoo once weekly is often recommended.
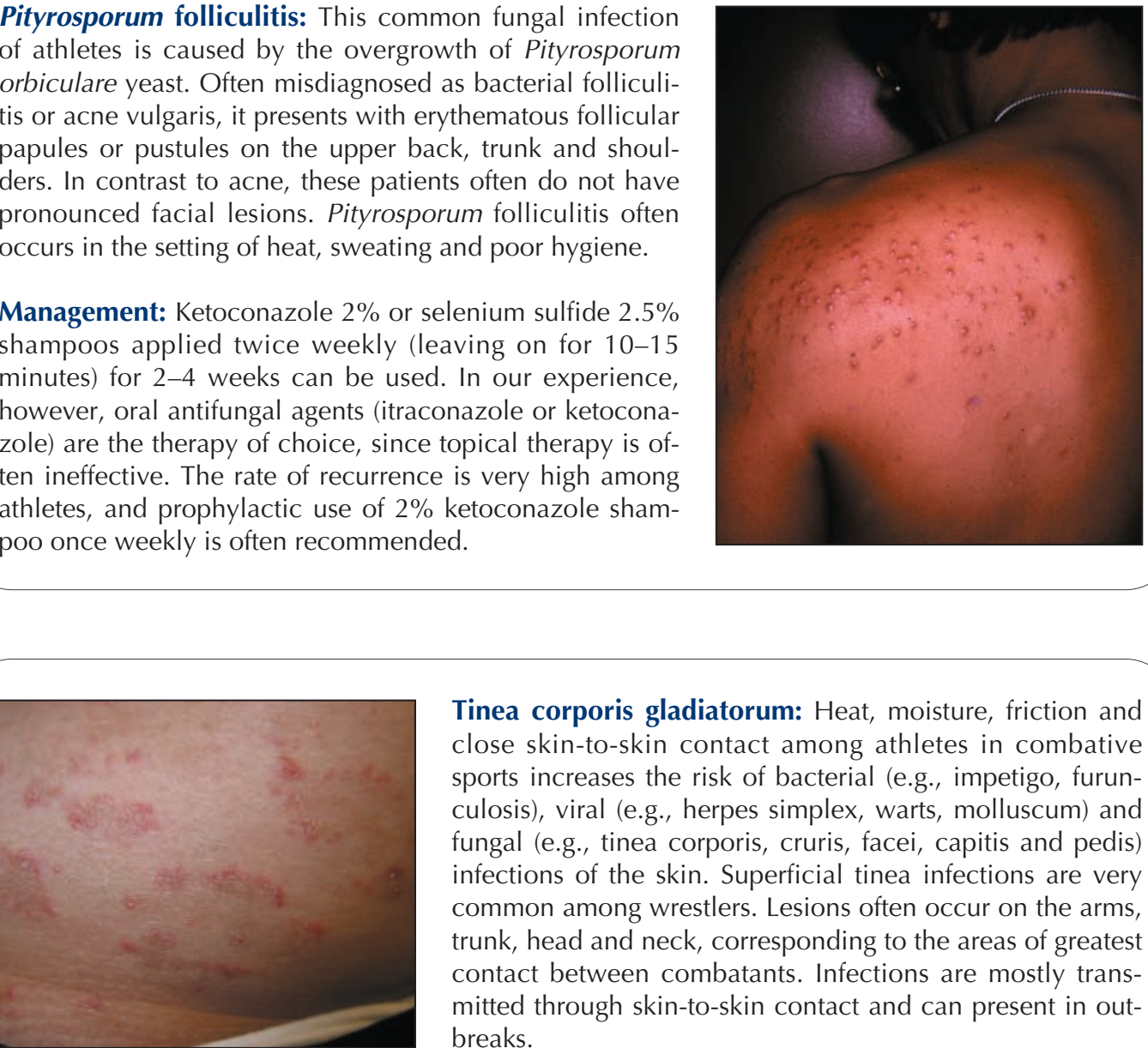

Tinea corporis gladiatorum: Heat, moisture, friction and close skin-to-skin contact among athletes in combative sports increases the risk of bacterial (e.g., impetigo, furunculosis), viral (e.g., herpes simplex, warts, molluscum) and fungal (e.g., tinea corporis, cruris, facei, capitis and pedis) infections of the skin. Superficial tinea infections are very common among wrestlers. Lesions often occur on the arms, trunk, head and neck, corresponding to the areas of greatest contact between combatants. Infections are mostly transmitted through skin-to-skin contact and can present in outbreaks.

Management: The recommended treatment of tinea gladiatorum is topical antifungal therapy (e.g., ketoconazole cream applied twice daily for 2-4 weeks). Indications for oral antifungal therapy include inflammatory lesions, extensive disease, tinea capitis and failed topical therapy. Prevention of transmission is paramount. If the lesions cannot be covered, athletes should be excluded from wrestling until healing occurs. Good hygiene practices (e.g., no towel sharing) should be advocated, ${ }^{3}$ and cleansing wrestling mats with bleach has been recommended by some.

Striae distensae or stretch marks: Intense sports, such as weightlifting, body building, football, wrestling and gymnastics, can result in continuous and progressive stretching of skin, which may lead to stretch marks. These can also occur during pregnancy, weight gain, rapid growth spurts, anabolic steroid use and as a result of Cushing's disease.

Management: The treatment of stretch marks is difficult. Although the evidence is not conclusive, topical tretinoin or laser therapy can potentially be effective. ${ }^{3}$

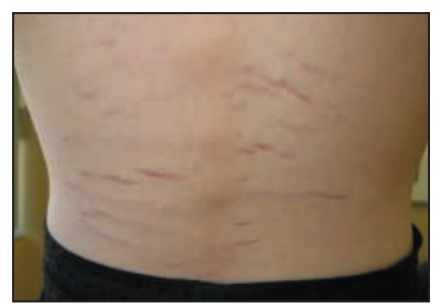

References

1. Knapik JJ, Reynolds K, Barson J. Influence of an antiperspirant on foot blister incidence during crosscountry hiking. 7 Am Acad Dermatol 1998;39(2 Pt 1):202-6.

2. Levine N. Dermatologic aspects of sports medicine. Dermatol Nurs 1994;6(3):179-86.

3. Adams BB. Dermatologic disorders of the athlete. Sports Med 2002;32 (5):309-21. 